EPiC Series in Engineering
Volume 3, 2018, Pages 931-935
HIC 2018. 13th International
Conference on Hydroinformatics

\title{
Assessment of surface moisture in the catchment area on the base of modelling the hydrological properties of soils.
}

\author{
Ilinich V.V. ${ }^{1}$, Bolotov A.G. ${ }^{1}$, Shein E.V. ${ }^{2}$, Makarychev S.V. ${ }^{3}$ \\ ${ }^{1}$ Russian state agrarian university - Moscow agricultural academy \\ ${ }^{2}$ Moscow State University named M.V. Lomonosov \\ ${ }^{3}$ Altai State Agrarian University \\ vilinitch@gmail.com
}

\begin{abstract}
.
The research is dedicated to estimation of soil moisture before storm rain flood for calculations of water erosion on the catchment. Modeling of hydrological properties of soils is used for characteristic of the soil moisture. The model based at technologies of multiple nonlinear regression, as well as the method of artificial neural networks.
\end{abstract}

\section{Introduction}

It is known that the most informative predictors of the model for forecasting flood characteristics are flood-forming precipitations and indicators of soil moisture of river basins before flood. So there is necessary to determine index of soil moisture using indirect indicators are used, including empirical coefficients (Bolotov, 2017), which to obtain experimentally often difficult. This information about moistening of the soil is important both for estimating the maximum water discharge and for estimating channel and planar erosion of soils.

Usually calculations of soil moisture are carried out on the basis of a hydrophysical analysis, which includes an assessment of water infiltration into the soil, moisture transfer and water retention. Majority of hydrologic modeling systems use hydraulic conductivity which provides estimates of this parameter as a function of texture class, it may be found from a soil survey (Hydrologic Modeling System HEC-HMS. Technical Reference Manual, 2000), (MIKE11 - a modelling System for River and Channels. Reference Manual, 2008). HEC HMS take consideration catchment soil but uses limited table of hydraulic conductivity (Hydrologic Modeling System HEC-HMS. Technical Reference Manual, 2000). However, when the land use changes the values of hydraulic conductivity differ from the tabulated ones. One of the methods for obtaining of hydrological parameters is the functional dependences (pedotransfer functions - PTF), which allow transforming information on 
basic soil properties into information about its transport characteristics (Bolotov, 2017), (Shein E.V., Dembovetsky A.V., Guber A.K., 1999), (Simunek J., van Genuchten M.Th., Sejna M., 2005).

In this paper, an approach is proposed for assessing soil moisture of the catchment area before flood runoff on the basis of modeling the hydrological properties of soils. These properties are used in mathematical models of moisture transfer in soils.

\section{Methods and materials}

To assess the erosion of the zonal soils of the catchment, synchronous with the measurement of the soil moisture pressure, the hydraulic conductivity was determined from the data on the centrifugation of kinetics - the dependence of change in sample moisture on time at a given centrifuge rotation speed (Smagin A.V., Sadovnikova N.B., Mizuri M.B.A., 1998) determined. The obtained hydraulic conductivity characterizes the ability of the soil to conduct a flow of moisture when changing soil water pressure.

The water retention $P=P(\theta)$ and the hydraulic conductivity $K=K(\theta)$ of unfrozen soil were calculated by the following formulas (van Genuchten, 1980):

$$
\theta=\theta_{r}+\frac{\theta_{s}-\theta_{r}}{\left[1+(\alpha P)^{n}\right]^{m}}, K=K_{s}\left(\frac{\theta-\theta_{r}}{\theta_{s}-\theta_{r}}\right)^{\frac{1}{2}}\left[1-\left[1-\left(\frac{\theta-\theta_{r}}{\theta_{s}-\theta_{r}}\right)^{\frac{1}{m}}\right]^{m}\right]^{2}, m=1-\frac{1}{n},
$$

where $\theta$ - volumetric soil moisture; $\theta_{s}$ and $\theta_{r}$ are the saturated and residual water contents respectively; $\alpha$ is the parameter, which is related to the inverse of the air entry pressure; $\mathrm{n}$ is the parameter, which is a measure of the pore-size distribution; $K_{s}-$ is the saturated hydraulic conductivity.

Modeling of the soil moisture regime was carried out using the predictive model Hydrus- $1 D$ (Simunek J., van Genuchten M.Th., Sejna M., 2005).

\section{Result and discussion}

We obtained our own continuous PTF for the characteristic soils of the Altai Region. The data set consisted of 810 experimental water retention curves and the hydraulic conductivity with simultaneous data on the particle size distribution, addition density and organic matter content. PTFs were constructed by training array, which is $2 / 3$ of the total sample size. The accuracy of the forecast was estimated from the test sample, which is $1 / 3$ of the total sample size.

A functional-parametric method was used in which regression dependencies relate the parameters of the water retention curve approximation to the basic soil properties. The predictors were: the addition density, the particle size distribution, the organic carbon content, and the parameters of the van Genuchten model (van Genuchten, 1980), (Simunek J., van Genuchten M.Th., Sejna M., 2005) of water retention as the response variable.

The methods of multiple linear and nonlinear regression were used, as well as the method of artificial neural networks (ANN) for creation a mathematical model describing the relationship between the values of hydrothermal parameters and the values of soil properties. At the same time, with the help of the Neural Networks Statistica program, their own ANNs were created. Before creating the neural network, the array of conjugate data of the filtration coefficient $\left(K_{s}\right)$ with the basic 
physical properties of the soil was divided into a learning set used for network training and a control set for testing the quality of the network operation.

The ratio of multiple nonlinear regression has been obtained with taking into account the nonlinear character of the dependence of the filtration coefficient $\left(K_{s}\right)$ on the soil properties:

$$
K_{s}=271,293+0,179 \cdot \text { Sand }-111,716 \cdot \operatorname{Ln}(\text { PhClay })+(1 / 267,203) \cdot \rho
$$

An analysis of standardized regression coefficients $(b)$ showed that the content of physical clay and the addition density (Table 1) make the greatest contribution to the regression of hydraulic conductivity for soils of the Altai Region.

Table 1. Characteristics of the standardized regression coefficients.

\begin{tabular}{|c|c|c|}
\hline parameter & $b$ & $b_{\text {StdErr }}$ \\
\hline Sand & 0,06 & 0,05 \\
\hline PhClay & $-0,67$ & 0,05 \\
\hline$\rho$ & 0,45 & 0,04 \\
\hline
\end{tabular}

The input parameters of the neural networks were the content of the sand fraction, physical clay, and the density of the soil. The smallest error in the calculation of $K_{s}$ was obtained using a neural network based on a radial-basis function with 3 inputs and 16 hidden neurons (Figure 1).

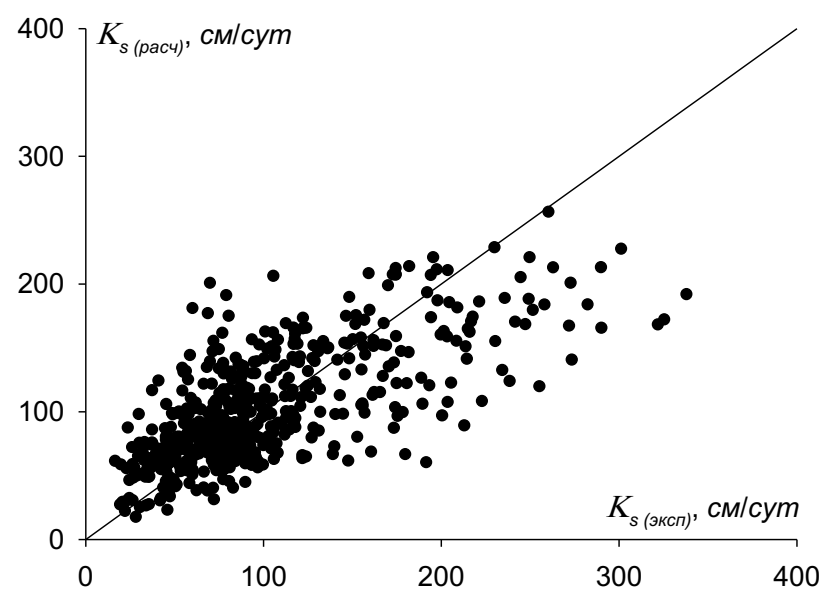

Figure 1. The correlation field of the calculated and experimental values of $K_{s}, S=36 \%$.

The influence of the change in the boundary conditions and hydrophysical parameters on the error in calculating the moisture transfer has been researched in order to assess the accuracy for determining these characteristics. For this, numerical experiments were performed with input parameters varying in the range from $-50 \%$ to $+50 \%$ relative to the initial values and recording the error in calculating the volumetric soil moisture (Figure 2). 

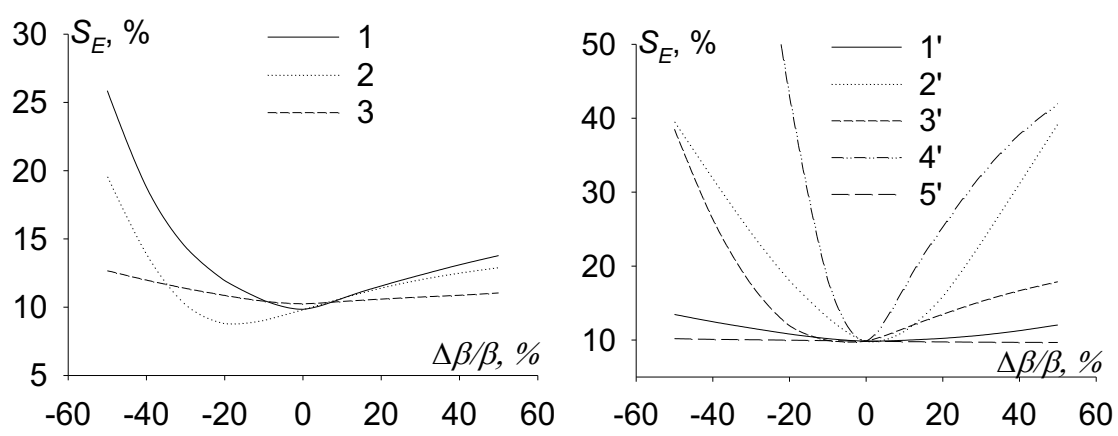

Figure 2. Dependence of the relative error in calculating the moisture content of the leached medium loamy chernozem $\left(S_{E}\right)$ on the change in the boundary conditions and the hydrophysical parameters: 1 - the upper boundary condition, 2 - the lower boundary condition, 3 - the initial condition; model parameters water retention of van Genuchten: $1^{\prime}-\theta_{r}, 2^{\prime}-\theta_{s}, 3^{\prime}-\alpha, 4^{\prime}-n, 5^{\prime}-$

$K_{s}$.

Analysis of the dependence of the relative error of the calculated soil moisture on the change in input parameters showed that the calculation error least depends on the change in the initial condition and parameter. For boundary conditions and the parameter this dependence is some what higher. The highest requirements for the accuracy of the specification of input quantities should be presented to the parameters of the model of water retention of van Genuchten, n, and, since the calculation error is greatest with the variation of these parameters. Figure 2 represents that the dependence shows the range of model adaptation. Also in the figure, it is seen, for the case considered, the possibility of adapting the model according to the lower boundary condition and parameter. The decrease of these values by $20 \%$ and $10 \%$, respectively, reduced the overall calculation error to $7.8 \%$. The criterion for model adaptation is the necessary and sufficient condition for the extrema (minimum) of the function $S_{E}=f(\Delta \beta / \beta): S_{E}^{\prime}(\Delta \beta / \beta)=0, S_{E}^{\prime \prime}\left(\Delta \beta_{0} / \beta_{0}\right)>0$, where $\Delta \beta_{0} / \beta_{0}$ - stationary point.

As a result of the study, it was revealed that among light loamy soils chestnut soils have the highest values of hydraulic conductivity, which is due to coarsely dispersed granulometric composition and a high number of large, well conducting moisture pores. The moisture pass through underlying horizons also quickly, that causes a good draining effect. The precipitates rapidly penetrate into the soil thickness, thereby virtually eliminating surface runoff. Among the medium- and heavyloamy soils, the highest values of $K_{r}$ have chernozems of the foothills and low mountains of the Altai. High values of the hydraulic conductivity of the upper horizons of the chernozems in this zone are due to good structuration, high porosity, which is able to pass moisture without hindrance, which ensures a rapid absorption of rains.

\section{Conclusions}

- A regional method for determining the soil moisture parameters is proposed for assessing the humidification of the catchment area before of the flood runoff.

- Soil hydraulic conductivity (reconstructed using pedotransfer functions) was obtained with a minimum error by the artificial neural network method according to the content of granulometric fractions and the addition density.

- The error in calculating the moisture content of the Altai chernozems is least dependent on the change in the initial condition and hydraulic conductivity. 


\section{Reference}

Bolotov, A. G. (2017). Hydrophysical state of soils of the southeast of Western Siberia. Moscow: Moscow State University named M.V. Lomonosov.

Hydrologic Modeling System HEC-HMS. Technical Reference Manual. (2000). Retrieved from http://www.hec.usace.army.mil/software/hec-hms/documentation/HEC-

HMS_Technical\%20Reference\%20Manual_\%28CPD-74B\%29.pdf

MIKE11 - a modelling System for River and Channels. Reference Manual. (2008). DHI Water Environment Health.

Shein E.V., Dembovetsky A.V., Guber A.K. (1999). Pedotransfer functions: obtaining, justifying and using. Eurasian Soil Science, 11, 1323-1331.

Simunek J., van Genuchten M.Th., Sejna M. (2005). The HUDRUS-1D Software Package for Simulating the One-Dimensional Movement of Water, Heart, and Multiple Solutes in VariablySaturated Media. Version 3.0. Riverside: Department of Environmental Sciences University of California.

Smagin A.V., Sadovnikova N.B., Mizuri M.B.A. (1998). Determination of the basic hydrophysical characteristics of soils by centrifugation method. Eurasian Soil Science, 11, 1362-1370.

van Genuchten, M. (1980). A Closed form equation for predicting the hydraulic conductivity of unsaturated soils. Soil Sci. Soc. Am. J. , 44, 892-898. 